\title{
Análisis dosimétrico de las incertidumbres derivadas del movimiento y las variaciones de densidad evaluadas con Radioterapia Guiada por la Imagen en Radioterapia Esterotáxica de pulmón
}

\section{Dosimetric analysis of uncertainties derived from movement and density variations evaluated with Image-Guided Radiotherapy in lung Stereotaxic Radiotherapy}

\author{
José Bea Gilabert, José Domingo Lago Martin, Enrique López Muñoz, Verónica González Vidal, \\ Paola Antonini Bolumburu, Miguel Ángel Berenguer Francés, Luis María Larrea Rabassa
}

Hospital Vithas Virgen del Consuelo. c/ Callosa d'En Sarrià 12 - 46007 Valencia.

Fecha de Recepción: 24/01/2020 - Fecha de Aceptación: 20/10/2020

Objetivo: Estudiar el impacto del movimiento y de la variación de la densidad en la dosis impartida al tumor analizando los volúmenes determinados sobre imágenes obtenidas durante el tratamiento.

Material y métodos: Se ha calculado la dosis de prescripción sobre el PTV (Planning Target Volume), considerando tanto una densidad uniforme forzada del mismo como sin forzarla, y el valor consecuente en el tumor. Se ha estudiado la diferencia entre los algoritmos Monte Carlo y Collapsed Cone. Se ha calculado la dosis sobre los volúmenes definidos en imágenes de cone beam durante el tratamiento, considerando idéntica densidad que el tumor. Finalmente, se han realizado dosimetrías teóricas sobre cilindros con diferentes desplazamientos desde el isocentro para evaluar su impacto en la dosis recibida comparada con los casos clínicos.

Resultados: La prescripción de dosis al PTV considerando una densidad forzada conduce a una dosis calculada mayor sobre el tumor de $10.6 \%$, y de $8.2 \%$ sin forzarla. Los volúmenes delimitados en imágenes de cone beam reciben aproximadamente igual dosis que el determinado en la planificación, con variaciones debidas a la incertidumbre de localización.

Conclusiones: La dosis calculada sobre volúmenes determinados sobre imagen cone beam obtenida durante el tratamiento permite reevaluar las condiciones dosimétricas asociadas a la prescripción.

Palabras clave: SBRT, IGRT, Heterogeneidad, Verificación de dosis.

Objective: To carry out an estimation in position and density variations by analysing volumes determined in cone beam images obtained during stereotactic radiotherapy lung treatments and its impact on delivered dose to tumour.

Materials and methods: Prescription dose for PTV (Planning Target Volume) and dose value obtained in tumour have been calculated, taking into account both forced density override of PTV and without forcing. Difference between Monte Carlo and Collapsed Cone algorithms has been studied. The treatment has been re-calculated on volumes generated by cone beam images, considering same density as tumour. Theoretical dosimetry has been carried out on cylinders with different shifts from isocenter in order to evaluate its impact on received dose comparing to other clinical cases.

Results: Prescription dose for PTV forcing density override leads to greater dose calculated on tumour of $10.6 \%$ and $8.2 \%$ without forcing. Volumes delineated on cone beam images receive approximately the same dose as the tumour determined in planning, dose differences being blamed to position uncertainty.

\footnotetext{
*Correspondencia: beagj@vithas.es

https://doi.org/10.37004/sefm/2020.21.2.003
} 
Conclusions: Dose calculated on volumes determined on cone beam images obtained during treatment could lead to prescription dose evaluation.

Key words: SBRT, IGRT, Heterogeneity, Dose verification.

\section{Introducción}

La Radioterapia Estereotáxica Extracraneal (SBRT, acrónimo en inglés de Stereotactic Body Radiation) se ha convertido en una herramienta fundamental en el tratamiento del cáncer de pulmón. 1,2 Esta patología presenta dos características específicas, como son el movimiento respiratorio y la heterogeneidad del medio circundante. En el primer caso, el movimiento respiratorio supone una dificultad añadida en la localización y delimitación de la lesión. Se han estudiado diferentes estrategias para abordar esta cuestión, ${ }^{3}$ recomendándose disponer de un sistema de control de la respiración, que puede estribar entre la compresión abdominal y el control respiratorio activo o gating respiratorio. Además, las modernas técnicas de tratamiento IMRT (Intensity Modulated Radiation Therapy) y VMAT (Volumetric Modulated Arc Therapy), basadas en haces estrechos, más el empleo de haces no filtrados FFF (Free Flattening Filter) con tasas altas de dosis, pueden presentar características especiales como son la alta modulación, ${ }^{4}$ que combinada con el movimiento del tumor pueden generar distribuciones de dosis muy heterogéneas sobre el mismo, lo cual es conocido como efecto interplay. ${ }^{5}$

La segunda característica específica corresponde al cálculo de dosis. El tumor está colindante a un tejido de baja densidad, bien como un nódulo inmerso en el pulmón, bien adyacente al mediastino o a la caja torácica. El informe ICRU-916 recomienda utilizar algoritmos tipo B como Collapsed Cone (CC) o Analitical Anisotropy Agorithm (AAA) y, sobre todo, Monte Carlo (MC), debido a que este último es el formalismo que mejor permite calcular el transporte de la radiación en interfases de medios heterogéneos. Además, debe contemplarse que el efecto de la heterogeneidad es más acusado con haces estrechos, produciéndose pérdida de equilibrio electrónico con el riesgo de infradosificaciones, y dificultando la optimización de la distribución dosis sobre el blanco.

La primera dificultad puede abordarse con los sistemas de imagen online de las unidades de tratamiento, que permiten monitorizar la posición del tumor verificándose que se está impartiendo la dosis sobre la lesión, es decir, el procedimiento de la IGRT (Image Guided Radiation Therapy). En particular, las imágenes CBCT (Cone Beam Computed Tomography) pueden ser utilizadas para determinar la posición del tumor antes y después del tratamiento permitiendo evaluar la esta- bilidad intrafracción. Sin embargo, no pueden utilizarse para calcular la dosis porque no contemplan todo el contorno del paciente. La segunda cuestión ha llevado a diferentes aproximaciones ${ }^{1}$ para soslayar este problema, como el cálculo dosimétrico considerando que el PTV (Planning Target Volume) tiene una Densidad Promedio Forzada (DPF) entre la lesión y el pulmón circundante para considerar un promedio de la densidad de los tejidos a lo largo del tratamiento; 7,8 sin embargo, otros autores presentan opiniones contrarias prefiriendo no forzar la densidad porque puede enmascarar el efecto de la pérdida de equilibrio electrónico. ${ }^{9,10}$

El objetivo de este trabajo es presentar una metodología para poder estimar la dosis impartida al GTV utilizando las imágenes de verificación online. ${ }^{11}$ Estudiaremos las desviaciones geométricas obtenidas y su relación con los márgenes obtenidos a priori, evaluando su impacto en la cobertura dosimétrica del GTV (Gross Target Volume). Se revisará el cálculo de dosis sobre el PTV, dependiente del uso o no de DPF y del algoritmo de cálculo utilizado. Evaluaremos la relación con la dosis calculada sobre el GTV planificado y con las dosis estimadas en los GTV determinados en las imágenes CBCT de cada sesión de tratamiento, de tal forma que podemos estimar la influencia del movimiento y las variaciones de densidad. Finalmente realizaremos una valoración sobre la posibilidad de revisar los criterios de evaluación de la dosis impartida sobre el GTV.

\section{Material y métodos}

\section{Exactitud de la colocación del paciente}

Se ha estudiado un total de 70 tratamientos de 3 sesiones, 10 tratamientos de 5 sesiones y 10 de una única sesión. Los pacientes se simularon en un CT Brightspeed (General Electric Healthcare, Chicago, EEUU) con técnica axial slow scan con periodo de rotación del tubo $T=4$ s y compresión abdominal sobre la guía Elekta BodyFIX (Elekta LTD, Estocolmo, Suecia). Se contornearon los volúmenes de tratamiento GTV con el sistema de planificación Elekta Monaco v5.11.2, estableciéndose un margen isótropo de $2 \mathrm{~mm}$ para el ITV, ${ }^{12}$ que aumenta hasta $5 \mathrm{~mm}$ para el PTV para contemplar la incertidumbre sistemática del acelerador y los dispositivos de adquisición de imagen. La planificación es transmitida al sistema de IGRT 
Elekta XVI v5.0.2, utilizándose como referencia para el registro de las imágenes CBCT obtenidas durante el tratamiento, siguiendo el protocolo específico de pulmón del Netherlands Cancer Institute NKI, ${ }^{*}$ donde se consideran rotaciones válidas menores de $3^{\circ}$ y se aplican únicamente desplazamientos. Las imágenes CBCT son adquiridas en una rotación completa de un minuto de duración para obtener una buena calidad de imagen. En cada sesión se realizan tres exploraciones: (1) una para realizar la corrección inicial de la posición y ajustarla al isocentro $\mathbf{r}_{1}$, (2) una verificación tras el desplazamiento de la mesa $\mathbf{r}_{2}$ y (3) otra al final de la sesión para evaluar la estabilidad del paciente $\mathbf{r}_{3}$. La verificación del desplazamiento con el segundo CBCT nos permite evaluar la estabilidad interfracción y mientras que la diferencia entre el CBCT final y éste $\mathbf{r}_{3}-\mathbf{r}_{2}$ nos indica la estabilidad intrafracción. A partir de los valores de cada paciente se obtienen las desviaciones de toda la población estudiada, ${ }^{13}$ tanto las sistemáticas $\Sigma$ como las aleatorias $\sigma$.

A continuación, se exporta el segundo CBCT al sistema de planificación ISOgrayv4.2.3 (Dosisoft SA, Cachan, Francia), transformando la matriz 3D a una sucesión de cortes CT con el mismo sistema de referencia y espesor que la simulación virtual. Sobre estas exploraciones se determinan los GTVi ${ }^{\mathrm{CBCT}}$ obtenidos durante la irradiación. Así mismo, para tratamientos de más de una sesión, se define el volumen UNI como la unión de los GTV iCBCT delimitados sobre los CBCTi de cada sesión de tratamiento, $U N I=\cup G T V i{ }^{C B C T}$. Este volumen se puede considerar como el valor del GTVm ${ }^{\mathrm{CBCT}}$ promedio más el margen aleatorio $\mathrm{M}$ de posición y de deformación, ${ }^{12}$ es decir, UNI = GTVm ${ }^{\mathrm{CBCT}}+\mathrm{M}$. De esta forma se puede estimar el Baseline Shift (BS) o diferencia de posición del GTV respecto al volumen planificado (ecuación 1), es decir,

$$
\mathbf{r}_{\mathrm{BS}}=\mathbf{r}_{\mathrm{GTVm}}^{\mathrm{CBCT}}-\mathbf{r}_{\mathrm{GTV}}
$$

que nos permite calcular el error sistemático $\Sigma_{\mathrm{BS}}$.

Se puede calcular entonces el margen $M$ siguiendo el formalismo de Sonke et al. ${ }^{14}$ Básicamente, se aplica la fórmula de Van Herk ${ }^{13}$ (ecuación 2)

$$
M=2.5 \cdot \Sigma+0.7 \cdot \sigma
$$

que contempla los valores de las desviaciones interfracción e intrafracción, tanto sistemáticas $\Sigma$ (ecuación 3) como aleatorias $\sigma$ (ecuación 4) sumadas en cuadratura. Además, incluye el valor sistemático $\Sigma$ de la delineación del GTV; en este caso, se considera que corresponde al valor de $\Sigma_{\mathrm{BS}}$ porque indica la diferencia entre el GTV

\footnotetext{
${ }^{*}$ XVI Protocols. NKI-AVL The Netherlands (2011).
}

delineado en la planificación con los obtenidos en cada sesión durante el tratamiento. El valor de $\sigma$ debería de contemplar la respiración, pero ésta ya estaría incluida en la determinación de GTVi ${ }^{C B C T}$, realizada a partir de una exploración de un minuto de duración englobando varios ciclos respiratorios. Es decir, dentro de la ecuación 2 se introduce

$$
\begin{aligned}
& \Sigma^{2}=\Sigma_{\text {interfracción }}^{2}+\Sigma_{\text {intrafracción }}^{2}+\Sigma_{\mathrm{BS}}^{2} \\
& \sigma^{2}=\sigma_{\text {interfracción }}^{2}+\sigma_{\text {intrafracción }}^{2}
\end{aligned}
$$

\section{Dosimetrías clínicas}

Tomando como base la población anterior, se ha estudiado un total de 44 pacientes, 32 de ellos tratados en tres sesiones, 2 en 5 sesiones y 10 en una única sesión. Se han escogido tratamientos realizados con un conjunto de 9 hasta 12 haces coplanares conformados (3DRT) en un acelerador Elekta Infinity con energía $6 \mathrm{MV}(\mathrm{Q} \mathrm{I}=0.679)$ y colimador Agility con láminas de 5 $\mathrm{mm}$ de anchura en el plano del isocentro. La dosimetría se ha calculado con el sistema de planificación Monaco y el algoritmo $X V M C^{15,16}$ que proporciona la dosis en medio (Dm), con una matriz $2 \mathrm{~mm}$ y varianza $1 \%$. Los campos se conformaron con un margen de $5 \mathrm{~mm}$ buscando el objetivo clínico de irradiar el 95\% del volumen del PTV (D95) con la dosis de prescripción.

Consideramos dos métodos de cálculo. En primer lugar, se asigna al PTV una densidad promedio $\left(\rho_{\mathrm{PTV}}\right)$ entre el GTV y el pulmón, lo cual definimos como método de cálculo forzando la densidad sobre el PTV (en adelante DF_PTV), calculándose la dosimetría del tratamiento, obteniéndose D95(PTV) XVMc $^{\text {DF_PTV }}=100 \%$ y evaluando D95(GTV) XVMc DF_PTV (fig. 1). A continuación, se recalcula sin forzar la densidad de ningún volumen, lo cual definimos como método sin forzar densidad (en adelante NF), para obtener el valor de D95(GTV) $\mathrm{XVMc}^{\mathrm{NF}}$. Ambas dosimetrías son enviadas al sistema de planificación ISOgray, siendo los planes recalculados en agua (Dw) con el algoritmo $\mathrm{CC}^{17}$ obteniéndose

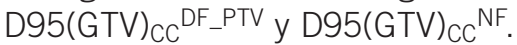

Con estos valores determinamos, en primer lugar, la diferencia de dosis calculada en el GTV, D95(GTV) del algoritmo CC respecto al algoritmo de referencia XVMC, para ambos métodos NF y DF_PTV. En segundo lugar, comparamos el método NF respecto al método DF_PTV de referencia, para ambos algoritmos XVMC y CC. Así mismo evaluaremos la diferencia global respecto a la dosis de prescripción D95(PTV) XVMc ${ }^{\text {DF_PTV }}$, para ambos métodos y ambos algoritmos.

A continuación, se realiza un cálculo adicional sobre el GTV exclusivamente con el algoritmo CC 


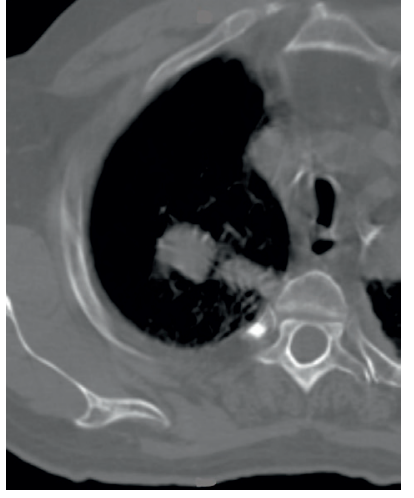

(a)

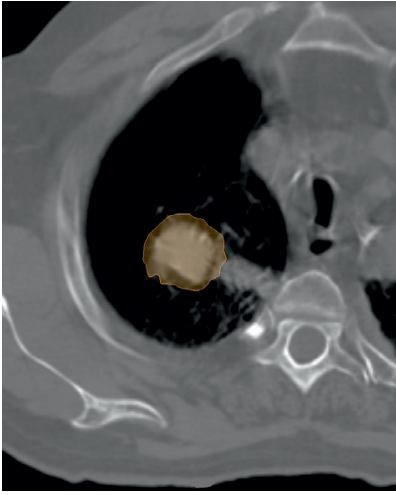

(b)

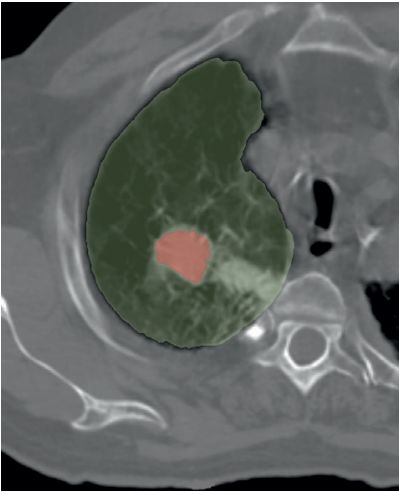

(c)

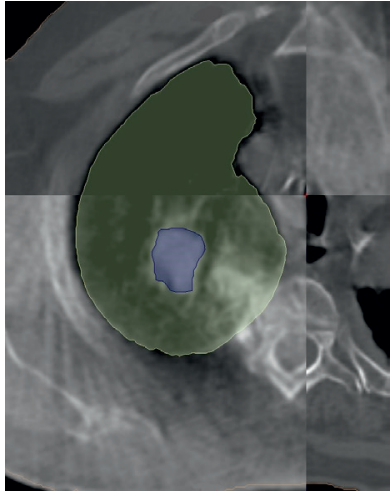

(d)

Fig. 1. Esquema de los métodos empleados: (a) NF, método sin forzar densidad (b) DF_PTV, método de cálculo forzando la densidad sobre el PTV, con densidad promedio entre el GTV y el pulmón (c) DF_GTV, método de cálculo forzando la densidad sobre el GTV, asignando sendas DPF tanto al GTV como al pulmón, (d) DF_CBCT, método de cálculo forzando la densidad sobre el GTV delineado en CBCT, asignando sendas DPF tanto al GTViCBCT como al pulmón.

asignando sendas DPF tanto al GTV ( $\left.\rho_{\mathrm{GTV}}\right)$ como al pulmón $\left(\rho_{\text {LUNG }}\right)$, lo cual definimos como método de cálculo forzando la densidad sobre el GTV (en adelante DF_GTV), obteniéndose D95(GTV) CC $^{\text {DFGTV }}$, de tal forma que nos sirva de referencia para analizar las imágenes de CBCT. Evaluaremos en este caso la diferencia respecto a los cálculos precedentes con ambos métodos, D95(GTV) CC $^{\text {DF_PTV y D95(GTV) }}{ }_{C C}{ }^{\text {NF. }}$.

Como el CBCT no contempla todo el volumen del paciente no puede utilizarse para calcular la dosis, y entonces los volúmenes GTViCBCT se exportan al CT de planificación. A continuación, se procede a recalcular la dosis con el algoritmo CC, forzando la densidad electrónica del GTV para cada GTVi ${ }^{\mathrm{CBCT}}$, $\rho_{\mathrm{GTV}}{ }^{\mathrm{CBCT}}=$ $\rho_{\mathrm{GTV}}$, así como la del pulmón circundante, es decir, método de cálculo forzando la densidad sobre el GTV delineado en CBCT (en adelante DF_CBCT). De esta forma, se obtiene una estimación de la interfase entre GTViCBCT y el pulmón circundante y de la dosis recibida por el GTV en cada sesión de tratamiento, $\mathrm{D} 95\left(\mathrm{GTV}{ }^{\mathrm{CBCT}}\right)_{\mathrm{CC}} \mathrm{CF}_{-}^{\mathrm{D}} \mathrm{CBCT}$. Estos valores se comparan con los valores calculados previamente sobre el GTV con los diferentes métodos, D95(GTV) ${ }_{C C}{ }^{\text {DF_PTV }}$, D95(GTV) ${ }_{C C}{ }^{\mathrm{NF}}$ y D95(GTV) CC $_{\text {DF_GTV }}$, así como con la dosis de prescripción D95(PTV) XVMC DF_PTV.

Finalmente, se ha tomado una muestra de 11 pacientes asignando al ITV una DPF entre el GTV y el pulmón ( $\rho_{\text {ITV }}$ ), lo cual definimos como método de cálculo forzando la densidad sobre el ITV (en adelante DF_ITV). Se ha repetido la dosimetría con el algoritmo

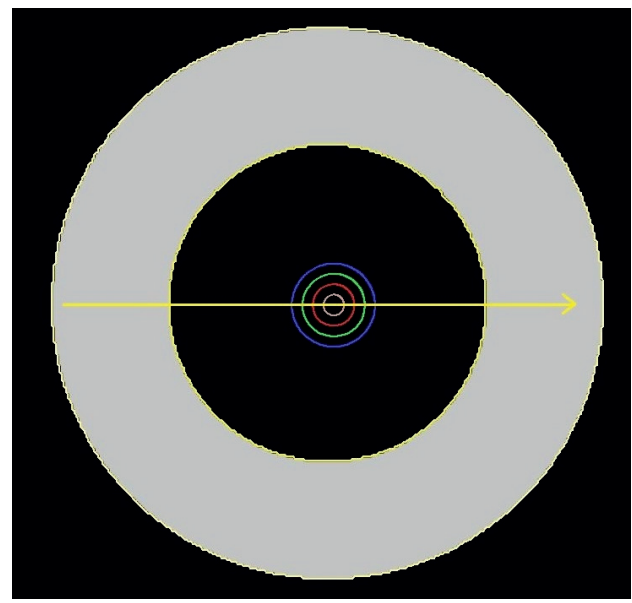

(a)

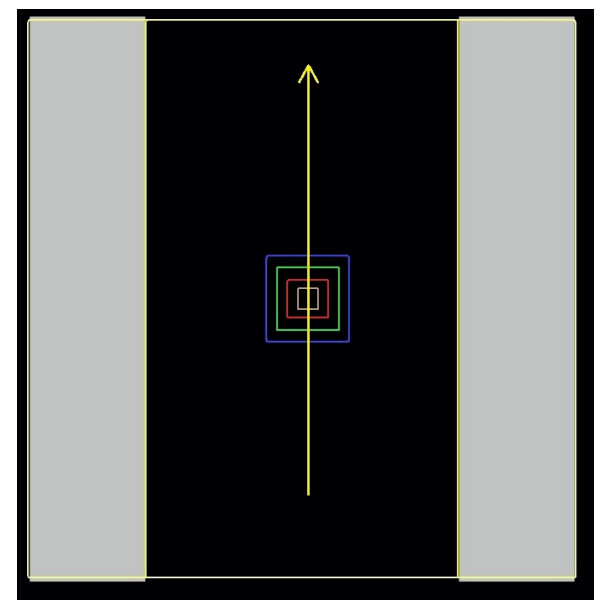

(b)

Fig. 2. Volúmenes cilíndricos, inmersos en cilindro equivalente pulmón, empleados en la dosimetría teórica: eje radial (a), eje longitudinal (b). 
CC conformando los campos sobre el ITV, de tal forma que $\mathrm{D} 95(\mathrm{ITV})_{\mathrm{CC}} \mathrm{DF}_{-} \mathrm{ITV}=100 \%$ de la dosis de prescripción y evaluando D95(GTV) ${ }_{C C_{D F} I T V}$, procediéndose a la misma comparación de ambas variables.

\section{Dosimetrías teóricas}

Se ha realizado una serie de dosimetrías teóricas en ambos planificadores para estudiar exclusivamente el efecto del tamaño y de los movimientos internos del GTV sobre su cobertura dosimétrica. Se han generado GTV de forma cilíndrica con radios de la base 5, 10, $15,20 \mathrm{~mm}$ y alturas iguales al doble del radio, centrados en un maniquí cilíndrico equivalente agua con un cilindro central con densidad de pulmón (fig. 2), que corresponde con la imagen CT virtual del detector Sun Nuclear Archeck (Sun Nuclear Corporation, Melbourne, EEUU). Se ha generado a su vez un ITV $=\mathrm{GTV}+2 \mathrm{~mm}$, así como varios $\mathrm{GTV}_{i}$, idénticos al GTV, cada uno desplazado una distancia $d_{i}$ respecto al isocentro, donde $i=1, \ldots, 5$ y $d_{i}=i \mathrm{~mm}$, en un caso sobre el eje radial y en otro caso en el longitudinal.

La dosimetría consiste en 12 haces que determinan una distribución de dosis cilíndrica sobre el ITV, aplicando DF_ITV $\left(\rho_{\text {ITV }}=0.5\right)$, de tal forma que D95(ITV) ${ }^{\text {DF_ITV }}=100 \%$ para ambos algoritmos. Este criterio es diferente del utilizado en los pacientes, donde se hace sobre el PTV, pero en este caso al tratarse de dosimetrías teóricas la incertidumbre sistemática de las unidades de diagnóstico y de tratamiento no debe ser contemplada. A continuación, se ha calculado D95 para cada uno de los GTV aplicando DF_GTV, para diferentes tamaños y posiciones $d_{i}$. Se han estudiado dos casos, un contraste alto entre GTV y pulmón (AC: $\rho_{\mathrm{GTV}}=1$, $\left.\rho_{\text {PULMON }}=0.2\right)$ y otro bajo $\left(\mathrm{BC}: \rho_{\mathrm{GTV}}=0.6, \rho_{\text {PULMON }}=\right.$ 0.4). En este caso, se ha evaluado la variación de la dosis D95(GTV) $\left(d_{i}\right)^{\text {DF_GTV }}$ al desplazarse una distancia $d_{i}$ respecto a la referencia D95(ITV) ${ }^{\text {DF_ITV }}$ para ambos algoritmos XVMC y CC.

\section{Resultados}

\section{Exactitud de la colocación del paciente}

La exactitud de la colocación de la población de pacientes se muestra la fig. 3 , mientras que en la tabla 1 se distingue entre tratamientos de 3 y 5 sesiones, indicándose las desviaciones ${ }^{13}$ promedio $\mu$, sistemática $\Sigma$ y aleatoria $\sigma$. Los resultados interfracción e interfracción están dentro de lo indicado en la bibliografía. ${ }^{1}$ Los valores de $\Sigma_{\mathrm{BS}}$, que indica la diferencia entre el GTV delineado en un CT convencional y en un CBCT son mayores que los correspondientes a las variabilidades

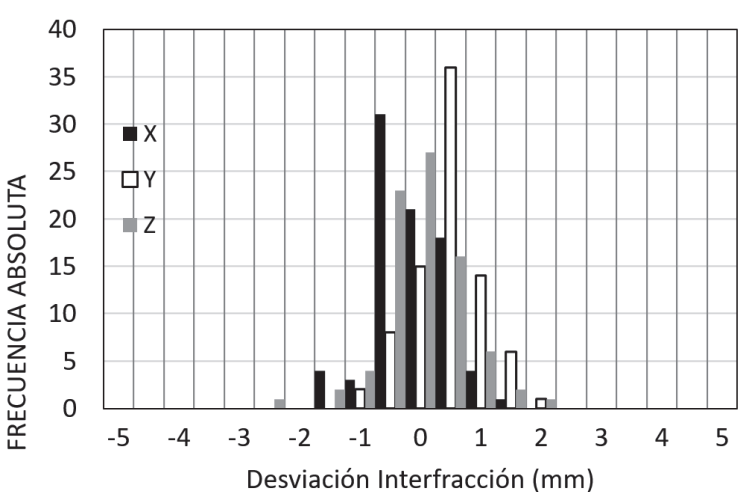

(a)

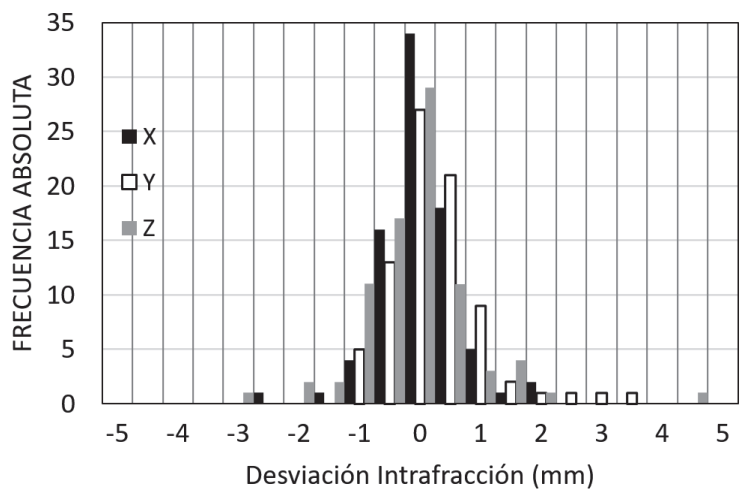

(b)

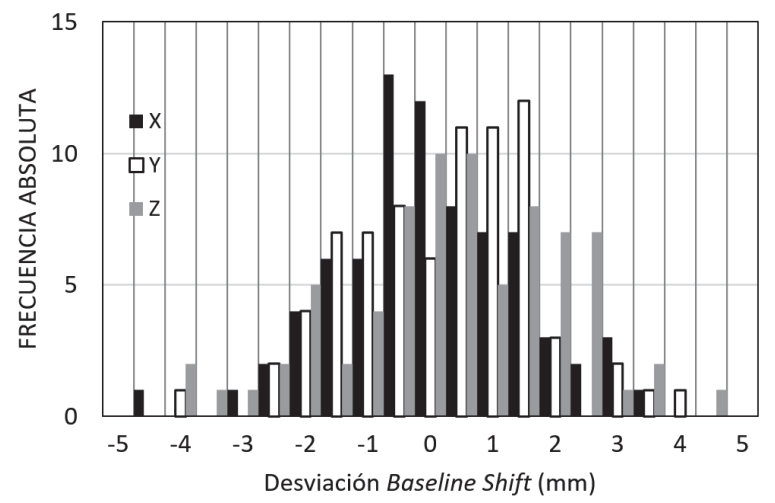

(c)

Fig. 3. Histogramas de las desviaciones interfracción (a), intrafracción (b) y BS (c) de la población total de pacientes estudiados. Los ejes siguen el convenio IEC.

interfracción e intrafracción. Los márgenes obtenidos siguiendo el formulismo de Sonke ${ }^{14}$ (ecuaciones 2, 3 y 4) nos dan resultados medios de $6 \mathrm{~mm}$, aunque para tratamientos de 5 sesiones alcanza $7.6 \mathrm{~mm}$ en el eje $\mathbf{Z}$ anteroposterior.

\section{Dosimetría clínica}

Los valores promedio de la densidad electrónica relativa a agua de son $\rho_{\text {PULMON }}=0.3 \pm 0.1, \rho_{\text {GTV }}=0.8$ $\pm 0.1, \rho_{\text {PTV }}=0.6 \pm 0.1$ en el conjunto de las dosimetrías evaluadas. En la tabla 2 se muestran los resultados estadísticos de las diferencias de dosis $\triangle$ D95(GTV), a 
Tabla 1. Desviaciones interfracción, intrafracción y BS promedio $(\mu)$, sistemática $(\Sigma)$ y aleatoria $(\sigma)$ de la población de pacientes estudiados, 70 tratados en 3 fracciones y 10 en 5 fracciones. Los márgenes se han calculado siguiendo el formalismo de la referencia 12).

\begin{tabular}{|c|c|c|c|c|c|c|c|c|c|}
\hline \multirow{2}{*}{\multicolumn{2}{|c|}{$\begin{array}{l}\text { Resultados }(\mathrm{mm}) \\
\text { Número sesiones }\end{array}$}} & \multicolumn{2}{|c|}{ Interfracción } & \multicolumn{2}{|c|}{ Intrafracción } & \multicolumn{2}{|c|}{ Baseline Shift } & \multicolumn{2}{|c|}{ Margen } \\
\hline & & 3 & 5 & 3 & 5 & 3 & 5 & 3 & 5 \\
\hline \multirow{3}{*}{$\mu$} & $x$ & -0.1 & -0.1 & 0.0 & 0.1 & 0.0 & 0.9 & 5.1 & 5.9 \\
\hline & Y & 0.5 & 0.2 & 0.2 & 0.2 & 0.1 & 0.4 & 5.9 & 4.3 \\
\hline & Z & 0.0 & -0.6 & -0.1 & 0.0 & -0.6 & -0.4 & 6.3 & 7.6 \\
\hline \multirow{3}{*}{$\Sigma$} & $x$ & 0.6 & 0.6 & 0.6 & 0.7 & 1.4 & 1.5 & & \\
\hline & Y & 0.6 & 0.7 & 1.1 & 0.3 & 1.5 & 1.0 & & \\
\hline & Z & 0.6 & 0.7 & 1.0 & 0.3 & 1.6 & 2.2 & & \\
\hline \multirow{3}{*}{$\sigma$} & $x$ & 0.9 & 1.5 & 1.1 & 1.5 & 0.9 & 1.0 & & \\
\hline & Y & 1.0 & 1.2 & 1.0 & 1.2 & 1.3 & 1.6 & & \\
\hline & Z & 1.2 & 1.8 & 1.5 & 1.7 & 1.7 & 1.7 & & \\
\hline
\end{tabular}

las cuales se les ha aplicado la Prueba de los Rangos con Signo de Wilcoxon. No se ha encontrado correlación entre $\Delta \mathrm{D} 95(\mathrm{GTV})$ y las densidades $\rho$ que sea estadísticamente significativa.

Los algoritmos XVMC y CC calculan dosis similares (fig. 4) con diferencias $0.0 \% \pm 2.4 \%$ con el método DF_PTV y $-0.2 \% \pm 3.0 \%$ sin forzar densidad. Las diferencias entre métodos (fig. 5) quedan bien patentes porque al forzar la densidad del PTV se obtienen dosis superiores que las obtenida sobre la simulación de forma usual, con diferencias similares $2.4 \% \pm 2.8 \%$ (XVMC) y $2.2 \% \pm 2.1 \%(C C)$.

Comparamos las dosis calculadas D95(GTV) -para ambos métodos y algoritmos- con la dosis de prescrip-

Tabla 2. Diferencias de dosis en el parámetro D95 evaluado sobre el GTV entre diferentes algoritmos y métodos de cálculo, así como respecto a la dosis de tratamiento, para una población de 44 pacientes. Se indica también el valor $p$ de significancia estadística según la Prueba de Wilcoxon.

\begin{tabular}{|c|c|c|c|c|}
\hline Diferencias D95 (\%) & & $\begin{array}{c}\text { Media } \pm \\
\text { Sigma }(\%)\end{array}$ & HO & $\mathrm{p}$-valor \\
\hline \multirow{2}{*}{ Algoritmo CC respecto algoritmo XVMC } & Método DF_PTV & $0.0 \pm 2.4$ & \multirow{2}{*}{$\mu \neq 0$} & 0.020 \\
\hline & Método NF & $-0.2 \pm 3.0$ & & 0.431 \\
\hline \multirow{2}{*}{ Método NF respecto método DF_PTV } & Algoritmo XVMC & $-2.4 \pm 2.8$ & \multirow{2}{*}{$\mu \geq 0$} & $<0.001$ \\
\hline & Algoritmo CC & $-2.2 \pm 2.1$ & & 0.090 \\
\hline \multirow{2}{*}{ Método DF_PTV respecto dosis de prescripción } & Algoritmo XVMC & $10.7 \pm 6.5$ & \multirow{4}{*}{$\mu \leq 0$} & $<0.001$ \\
\hline & Algoritmo CC & $10.6 \pm 6.3$ & & $<0.001$ \\
\hline \multirow{2}{*}{ Método NF respecto dosis de prescripción } & Algoritmo XVMC & $8.3 \pm 6.2$ & & $<0.001$ \\
\hline & Algoritmo CC & $8.1 \pm 6.0$ & & $<0.001$ \\
\hline \multirow{2}{*}{$\begin{array}{l}\text { Método DF_GTV respecto métodos DF_PTV y } \\
\text { NF }\end{array}$} & Método DF_PTV & $0.8 \pm 2.8$ & \multirow{2}{*}{$\mu \leq 0$} & 0.029 \\
\hline & Método NF & $3.3 \pm 4.1$ & & $<0.001$ \\
\hline \multirow{3}{*}{$\begin{array}{l}\text { Método DF_CBCT respecto métodos DF_GTV, } \\
\text { DF_PTV y NF }\end{array}$} & Método DF_GTV & $-1.0 \pm 1.9$ & $\mu \geq 0$ & $<0.001$ \\
\hline & Método DF_PTV & $-0.2 \pm 3.3$ & $\mu \geq 0$ & 0.491 \\
\hline & Método NF & $2.1 \pm 4.0$ & $\mu \leq 0$ & 0.007 \\
\hline $\begin{array}{l}\text { Método DF_CBCT respecto dosis de } \\
\text { prescripción }\end{array}$ & - & $10.4 \pm 6.4$ & $\mu \leq 0$ & $<0.001$ \\
\hline
\end{tabular}




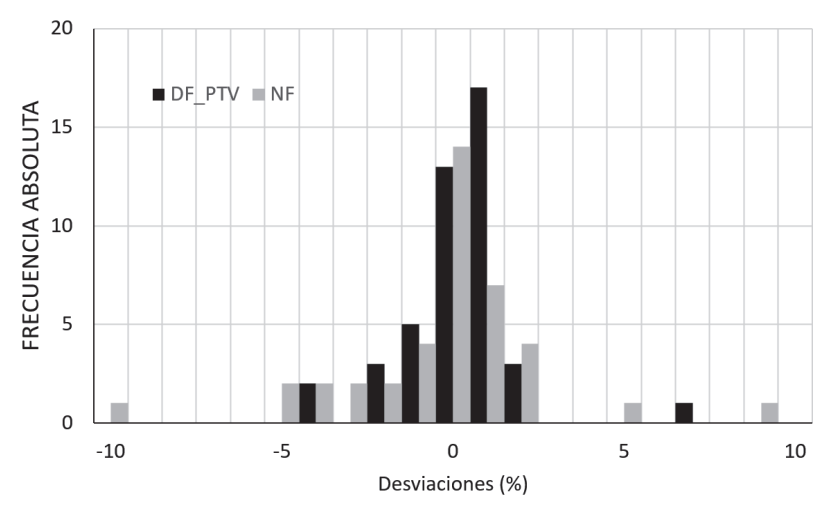

Fig. 4. Histograma de desviaciones del algoritmo CC respecto a XVMC en el parámetro D95 evaluado sobre el GTV, presentados para el cálculo sin forzar la densidad (NF) y forzando la densidad sobre el PTV (DF_PTV).

ción del tratamiento observándose que en todos los casos el objetivo de irradiación se cumple, en general calculándose una dosis mayor media $+10.8 \%$ con el método DF_PTV y $+8.5 \%$ con el método NF, aunque existen diferencias de hasta el $30 \%$.

Introducimos ahora el método DF_GTV con el algoritmo CC (fig. 6), donde $\mathrm{D} \mathrm{CCC}^{\mathrm{DF}} \bar{G}_{\mathrm{GTV}}(\mathrm{GTV})$ es prácticamente idéntica que DF_PTV $(+0.8 \% \pm 2.8 \%)$ y $3.3 \% \pm 4.1 \%$ mayor que el método NF, en concordancia con los resultados anteriores. Análogamente se ha aplicado sobre GTViCBCT el método DF_CBCT. La comparación con los otros tres métodos (fig. 7) indica resultados consistentes, en particular prácticamente igual que en el método DF_PTV $(-0.2 \% \pm 3.3 \%)$, pero un poco inferior que DF_GTV $(-1.0 \pm 1.9 \%)$. Finalmente, la diferencia $\mathrm{D} 95\left(\mathrm{GTV}_{i}{ }^{\mathrm{CBCT}}\right)_{\mathrm{CC}}{ }^{\mathrm{DF}}{ }^{\mathrm{CBCT}}$ respecto a la dosis de prescripción es $10.4 \% \pm 6.4 \%$, con un valor mínimo de $2 \%$.

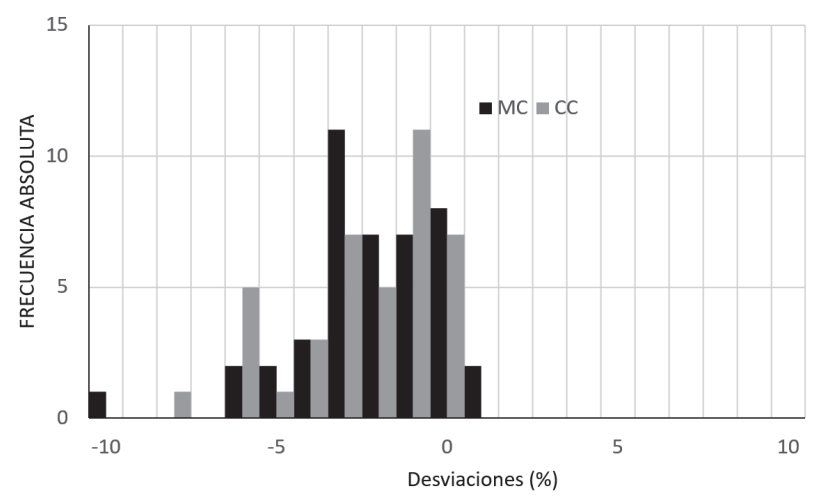

Fig. 5. Histograma de desviaciones del método NF respecto al método DF_PTV en el parámetro D95 evaluado sobre el GTV, presentados para los algoritmos de cálculo XVMC y CC.

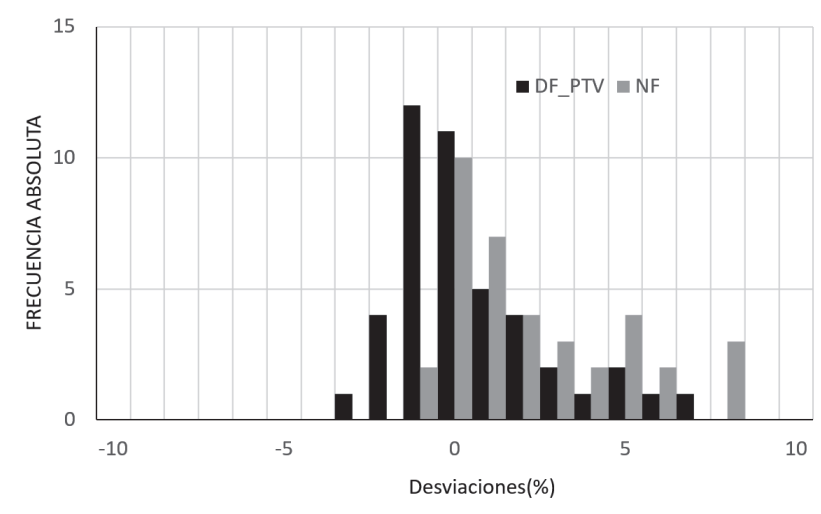

Fig. 6. Histograma de desviaciones en el parámetro D95 evaluado con el algoritmo CC sobre el GTV forzando su densidad (DF_GTV) respecto a los cálculos sin forzar la densidad (NF) y forzando la densidad sobre el PTV (DF_PTV).

Las dosimetrías teóricas conformadas al ITV nos da como resultado D95(GTV) ${ }_{C C}{ }^{\text {DF_ITV }}$ cuya diferencia respecto a la dosis de prescripción es $6.8 \pm 5.2 \%$, con un valor mínimo de $0 \%$, es decir, todos los valores están por encima de la dosis prescrita al ITV.

\section{Dosimetría teórica}

Se ha tomado como valor de referencia D95(ITV) DF_ITV $=100 \%$ de la dosis de prescripción. Se ha calculado la variación de D95(GTV) desplazándolo una distancia $d_{i}=1, \ldots, 5 \mathrm{~mm}$ desde isocentro, siendo negativos en la dirección longitudinal del eje del cilindro, mientras que los positivos indican la dirección radial. En casi todos los casos, se cumplen los objetivos de irradiación

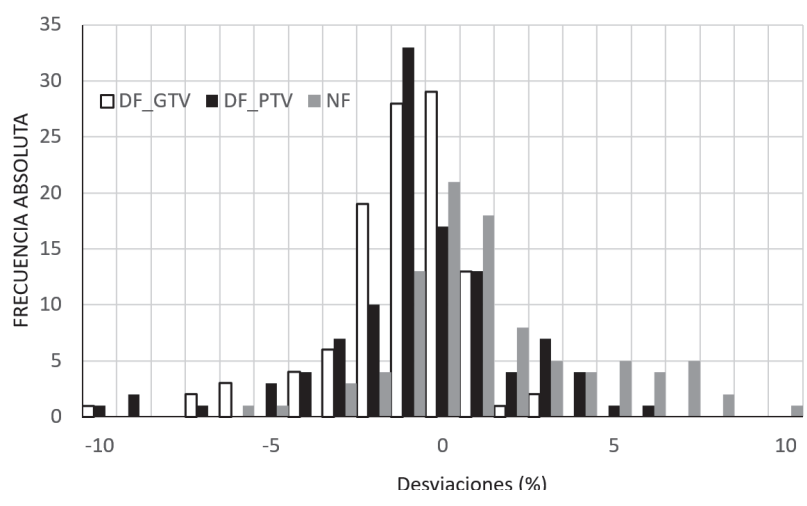

Fig. 7. Histograma de desviaciones en el parámetro D95 evaluado con el algoritmo CC sobre el GTV ${ }_{i}{ }^{\mathrm{CBCT}}$ forzando su densidad (DF_CBCT) respecto a los cálculos sobre el GTV sin forzar la densidad (NF), forzando la densidad promedio sobre el PTV (DF_PTV) y forzando la densidad sobre el propio GTV (DF_GTV). 

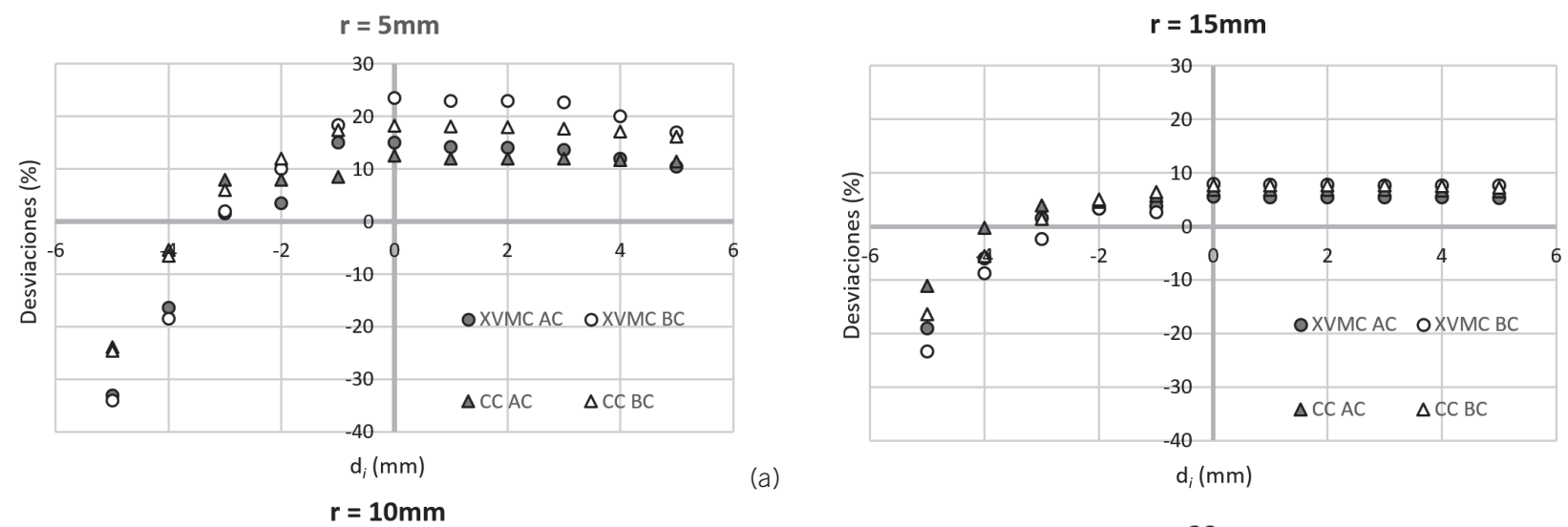

(a)

$\mathrm{d}_{i}(\mathrm{~mm})$

(c)
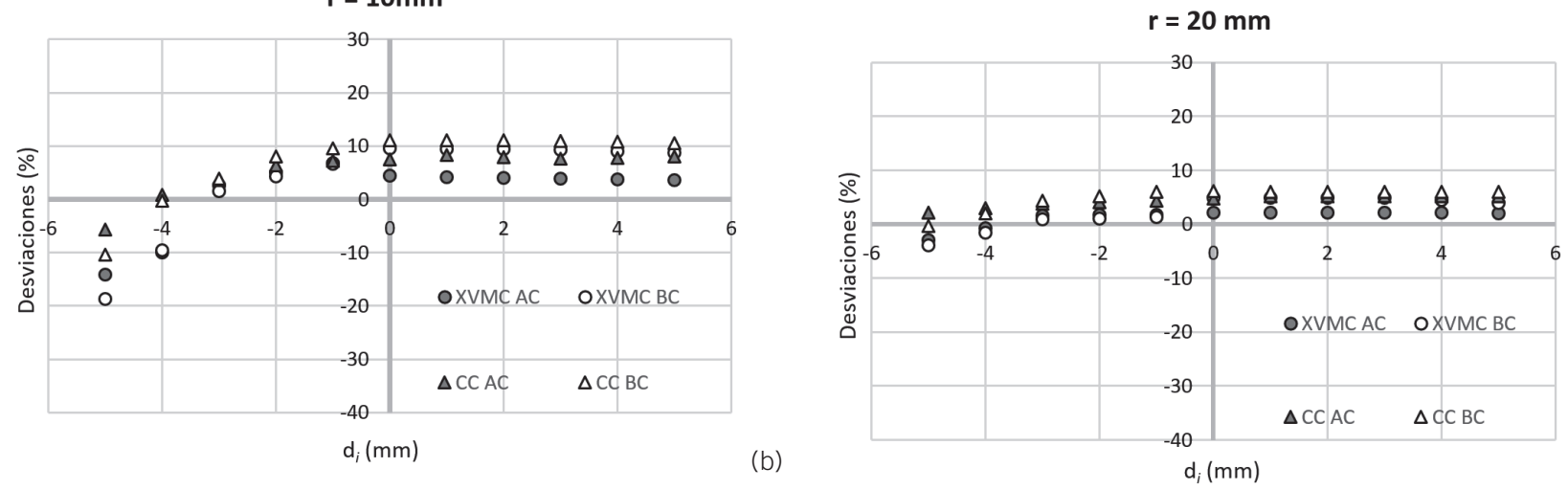

(d)

Fig. 8. Dosimetrías teóricas. Diferencias de la dosis D95 evaluada sobre el GTV desplazado una distancia $d_{i}$ respecto al isocentro calculada con el método DF_GTV con respecto al valor de referencia D95 evaluado sobre el ITV calculada con el método DF ITV. Se distinguen los algoritmos XVMC y CC y los casos estudiados alto contraste (AC) y bajo contraste (BC) de densidad. Se consideran cilindros con radio de la base (a) $5 \mathrm{~mm}$, (b) $10 \mathrm{~mm}$, (c) $15 \mathrm{~mm}$ y (d) 20 mm. Los desplazamientos $d_{i}$ negativos corresponden al eje longitudinal del cilindro, mientras que los positivos corresponden al eje radial.

de D95(GTV) respecto a la referencia D95(ITV). De hecho, la dosis que cubre el 95\% del volumen del GTV es un $10 \%$ mayor, y hasta un $20 \%$ cuando el radio es $r=5 \mathrm{~mm}$. Sólo se obtiene infradosificación para desplazamientos mayores que $3 \mathrm{~mm}$ en la dirección longitudinal.

Por otra parte, hay que indicar que en general la dosis calculada con bajo contraste es mayor que con alto contraste. En particular, con el algoritmo XVMC se obtienen diferencias de $2 \%$ para radio de $20 \mathrm{~mm}$ y hasta $7 \%$ para radio $5 \mathrm{~mm}$, en el isocentro y desplazándose sobre el eje radial, tendencia que se invierte para desplazamientos longitudinales mayores que $2 \mathrm{~mm}$.

\section{Discusión}

El reposicionamiento del paciente en cada sesión de tratamiento se realiza utilizando la guía BodyFIX y la verificación con imagen CBCT. La reproducibilidad del paciente queda reflejada en los valores de $\Sigma$ y $\sigma$ interfracción e intrafracción, que son del mismo orden que las referencias consultadas. ${ }^{1}$ La diferencia BS (ecua- ción 1) procede del hecho de que estamos registrando a estructuras subrogadas como el pulmón en vez de registrar a tumor. La otra razón estriba en que tenemos un CBCT con características de adquisición diferentes de las del CT de simulación, y en el que englobamos un minuto de respiración frente a 4 segundos, ambos con compresión abdominal en respiración libre. En consecuencia, sería más correcto hablar de un ITV que de un GTV en CBCT, ya que en la delineación de éste estaría incluido su movimiento debido a la respiración. En definitiva, existen diferencias entre la forma y posición del GTV determinados en el CT de planificación y el CBCT de verificación. Además, el contraste de densidad entre el GTV y pulmón va a ser mayor en la realidad que en $\mathrm{CBCT}$, pero no podemos determinarlo con precisión sin la existencia de un 4DCT.

Los valores de $\sum$ y $\sigma$ obtenidos para BS son mayores que los obtenidos en interfracción e intrafracción. Aplicando la fórmula de Van Herk según el formalismo de Sonke ${ }^{13,14}$ (ecuaciones 2, 3 y 4), donde ya está incluida la penumbra, el margen que debería de aplicarse al GTV Ilegaría hasta casi $8 \mathrm{~mm}$ en la dirección vertical para tratamientos de 5 sesiones. En nuestro 
caso el margen aplicado es de $2 \mathrm{~mm}$ al ITV añadiendo $3 \mathrm{~mm}$ más para definir el PTV. Ambos valores no son directamente comparables, porque nuestro método no contempla la penumbra y debemos añadir un margen de conformación de $5 \mathrm{~mm}$ al PTV, mientras que Sonke no contempla la incertidumbre sistemática del acelerador y el CT de simulación. Contemplando estas diferencias, ambos valores serían del mismo orden.

El margen de conformación evita el efecto gradiente $^{18}$ donde el GTV se desplaza fuera del volumen irradiado; evidentemente, márgenes de conformación menores que puedan exigir isodosis de prescripción diferentes pueden conducir a diferentes resultados. Los resultados dosimétricos apuntan en esa dirección ya que no se ha encontrado infradosificaciones en D95 $\left(\mathrm{GTV}_{i}{ }^{\mathrm{CBCT}}\right)_{\mathrm{CC}}{ }^{\mathrm{DF}} \mathrm{CBCT}^{\mathrm{CBC}}$, principalmente porque los valores de BS son menores que los márgenes utilizados. Por otra parte, debe recordarse que la técnica de tratamiento considerada en este estudio es 3DRT sobre GTV de forma convexa, por lo que hay que tener especial cuidado en técnicas moduladas, sobre todo sobre GTV con formas complejas con órganos de riesgo adyacentes, donde este formalismo debe ser reevaluado.

Los cálculos de la prescripción de tratamiento se han realizado con el algoritmo de Monte Carlo (Monaco $X V M C$ ) que calcula dosis en medio $D m$, y se han verificado con otro algoritmo tipo B, CC (Dosisoft ISOgray) que calcula la dosis en agua $D w$ puestos en escala por la densidad electrónica del medio. A las diferencias de dosis se les ha aplicado la Prueba de los Rangos con Signo de Wilcoxon, de tal forma que los resultados confirman nuestras hipótesis de partida, es decir, que los valores de media y desviación estándar de las diferencias de dosis son estadísticamente significativos. Por tanto, los valores numéricos de ambas dosis calculadas son equiparables (fig. 4) tal y como se indica en la literatura para tejidos blandos, ${ }^{19-21}$ por lo que CC puede utilizarse para la evaluación de la dosis impartida, que es calculada previamente con el algoritmo XVMC. Otros autores, como Rana et al., ${ }^{22}$ comparan de forma similar los algoritmos Acuros XB y AAA, encontrando que este último calcula valores de dosis D100 hasta un 5\% mayores, pero los autores no especifican si calculan la dosis en medio o en agua.

Se han realizado las dosimetrías al paciente forzando para el PTV una densidad electrónica promedio entre tumor y pulmón DF_PTV. Algunos autores lo recomiendan para mejorar la optimización, 7,8 pero otras referencias utilizan el CT de simulación sin DPF porque puede enmascarar el efecto de la pérdida de equilibrio electrónico. 9,10 Nuestros resultados indican que las dosis calculadas D95 DF_PTV son 2.3\% mayores que $\mathrm{D} 95^{\mathrm{NF}}$ con ambos algoritmos, ya que el aumento virtual de la densidad en la intersección del pulmón con el PTV permite alcanzar más rápidamente el equi- librio electrónico que utilizando el CT sin manipular. Solamente existen dos puntos fuera de la tendencia, que una vez revisados correspondieron a tumores cavitados, que presentan un alto contraste de densidad dentro del propio volumen. Debemos indicar que el trabajo de Archibald-Heeren ${ }^{23}$ et al. indica el efecto contrario, pero en este caso los desplazamientos estudiados llegan hasta $20 \mathrm{~mm}$. Finalmente, los valores D95(GTV) ${ }^{\text {DF_PTV }}$ son $10.6 \%$ mayores que la dosis prescrita, siendo D95(GTV) ${ }^{\mathrm{NF}}$ un $8.2 \%$ mayor, que confirma que el forzado de la densidad da como resultado un valor mayor de la dosis calculada sobre el GTV. En ambos casos no se han observado diferencias significativas entre nódulos inmersos en el pulmón o adyacentes a costillas o mediastino.

Se ha procedido a calcular la dosis sobre el GTV con el método DF_GTV, y la hemos comparado con los métodos previos. El valor medio de la diferencia $\Delta \mathrm{D} 95$ (GTV) DF_GTV es $0.8 \%$ mayor que el obtenido con el método de cálculo DF_PTV, y 3.3\% mayor que el método NF, en concordancia con los resultados anteriores. En consecuencia, es válido para comparar con la dosis calculada para GTVi delineado en el CBCT en cada sesión, que nos da como resultado D95(GTVi $\left.{ }^{\mathrm{CBCT}}\right)_{\mathrm{CC}}{ }^{\mathrm{DF}}{ }_{-} \mathrm{CBCT} 1.0 \%$ menor, debida básicamente a variaciones de posición y forma. Comparando con los otros métodos, es igual al método DF_PTV, además de un 2.1\% mayor que el cálculo con el método NF, diferencias consistentes con las obtenidas previamente. La solidez de estos resultados permite aplicar el método DF_CBCT para evaluar la dosis de los GTV $i$ definidos durante el tratamiento, independientemente de que utilicemos los métodos DF_PTV o NF. El resultado final es que las dosis estimadas D95(GTV $\left.{ }^{\mathrm{CBCT}}\right)_{\mathrm{CC}} \mathrm{CF}_{-} \mathrm{CBCT}$ son $10.4 \%$ mayores que las prescritas sobre el PTV, lo cual indica que los márgenes geométricos no se deberían de disminuir porque se demuestra experimentalmente con este procedimiento que garantizan la irradiación de los tumores. Finalmente, esta evaluación de la dosis en el GTV podría contemplarse en una perspectiva más amplia: por ejemplo, la Planificación Robusta 24 no considera el concepto de PTV, sino que optimiza la dosis al GTV contemplando el comportamiento aleatorio de su movimiento. En consecuencia, resultaría interesante la evaluación de dosis impartida al GTV en relación con los resultados clínicos con ambas metodologías.

Se han realizado dosimetrías teóricas conformando $5 \mathrm{~mm}$ de margen al ITV generado a partir de GTV de forma cilíndrica; de esta forma no se contempla el margen del PTV, que contempla la incertidumbre sistemática del acelerador, el XVI y la simulación. La irradiación calculada con CC es mayor que con XVMC, salvo para tumores pequeños; esto es debido a que el algoritmo XVMC modela mejor la dosis en las zonas de interfase, cuya proporción de volumen respecto al GTV es mayor para los tumores pequeños. El cálculo de los GTV nos 
da resultados de dosis mayores que la prescrita al ITV, salvo en el eje longitudinal para cilindros menores de $20 \mathrm{~mm}$ desplazados más de $3 \mathrm{~mm}$ tal y como se ve en la fig. 8. El eje longitudinal es más crítico que el radial, porque para idénticos desplazamientos el porcentaje de volumen fuera de la isodosis de tratamiento es mayor; de cualquier forma, se trataría del peor escenario, lejos de la forma irregular de los volúmenes de tratamiento. Además, los resultados estadísticos de los desplazamientos de los GTV $_{i}{ }^{\mathrm{CBCT}}$ (tabla 1) están por debajo de $3 \mathrm{~mm}$. Una muestra aleatoria de once pacientes se recalculó con el algoritmo CC siguiendo este criterio y en todos ellos los GTV $_{i}{ }^{\text {CBCT }}$ cumplieron los objetivos de irradiación.

Por otra parte, debe indicarse que los resultados teóricos indican que, para una misma densidad promedio del ITV, la dosis calculada sobre el GTV aumenta cuanto menor es el contraste entre tumor y pulmón, debido a que disminuye el efecto build up. Los volúmenes contorneados $\mathrm{GTV}_{i}{ }^{\mathrm{CBCT}}$ son en realidad un ITV con densidad promedio debido a las características de adquisición de la imagen CBCT, es decir, en la realidad el contraste sería mayor y la irradiación del GTV sería ligeramente menor. El análisis realizado sobre las dosimetrías de la población de pacientes no muestra relación entre la dosis calculada y el contraste de densidades, por lo que no se puede dar una estimación numérica de este efecto.

\section{Conclusiones}

La dosis calculada sobre el GTV es siempre mayor que la dosis prescrita al PTV, independientemente del algoritmo de cálculo. De la misma forma, la utilización de densidad promedio forzada puede ser útil durante el proceso de optimización del cálculo, pero la dosis final al GTV puede calcularse con el CT sin forzar densidad.

La determinación de estructuras sobre las imágenes de CBCT realizadas para verificar la posición del paciente nos permiten estimar la dosis recibida por el GTV, que en los casos estudiados siempre es mayor que la prescrita. En consecuencia, la definición del PTV y la conformación de los haces garantizan la irradiación del GTV dentro de los márgenes geométricos estudiados. Estudios complementarios al presente podrían aportar más información sobre la dosis impartida al GTV durante el tratamiento y su impacto en el control tumoral.

\section{Agradecimientos}

Los autores agradecen a Lidia Ros, Patricia Gil, Lorena Delgado y María López, técnicos superiores en dosimetría del Departamento de Radiofísica, su cuida- dosa y sistemática recopilación de los datos utilizados en la elaboración de este trabajo.

\section{Referencias}

1. Fernández Letón P, Baños Capilla MC, Bea Gilabert J, Delgado Rodríguez JM, De Blas Piñol R, Martínez Ortega J et al: Recomendaciones de la Sociedad Española de Física Médica (SEFM) sobre implementación y uso clínico de radioterapia estereotáxica extracraneal (SBRT) Rev Fis Med 2017;18(2):77-142 https://revistadefisicamedica.es/index. $\mathrm{php} / \mathrm{rfm} / \mathrm{article} / \mathrm{view} / 247$

2. Benedict SH, Yenice KM, Followill D, Galvin JM, Hinson W, Kavanagh B, et al. Sterotactic body radiation therapy: The report of AAPM Task Group 101, Med Phys 2010;37(8):4078-101. https://doi.org/10.1118//1.343808

3. Keall PJ, Mageras GS, Balter J, Emery RS, Forster K, Jiang $S$ et al. The management of respiratory motion in radiation oncology report of AAPM Task Group 76. Med Phys 2006;33(10):3874-900. https://doi.org/10.1118//1.2349696

4. IAEA-TRS-483 Dosimetry of Small Static Fields used in External Beam Radiotherapy. An IAEA-AAPM International Code of Practice for Reference and Relative Dose Determination 2017.

5. Yu CS, Jaffray DA, Wong JW. The effects of intra-fraction organ motion on the delivery of dynamic intensity modulation, Phys Med Biol 1998;43(1):91-104 https://doi. org/10.1088/0031-9155/43/1/006

6. ICRU Report No. 91 Prescribing, recording and reporting of stereotactic treatments with small photon beams, Journal of the ICRU Volume 14 No 22014.

7. Wiant D, Vanderstraeten C, Maurer J, Pursley J, Terrel J, Sintay BJ, On the validity of density overrides for VMAT lung SBRT planning, Med Phys 2014;41(8):081707. https://doi. org/10.1118/1.4887778

8. Zvolanek K, Ma R, Zhou C, Liang X, Wang S, Verma V et al., Still equivalent for dose calculation in the Monte Carlo era? A comparison of free breathing and average intensity projection CT datasets for lung SBRT using three generations of dose calculation algorithms, Med Phys 2017;44(5):1939-47 https://doi.org/10.1002/mp.12193

9. Disher B, Hajdok G, Gaede S, Battista JJ, An in-depth Monte Carlo study of lateral electron disequilibrium for small fields in ultra-low density lung: implications for modern radiation therapy, Phys Med Biol 2012;57(6):1543-59. https://doi. org/10.1088/0031-9155/57/6/1543

10. Qin Y, Chetty I, The Impact of Density Overrides in IMRT and VMAT Planning for Lung Cancer Patients Treated SBRT. En: AAPM 58th Annual Meeting \& Exhibition. Washington, DC; 2016. Presentation SU-F-T-543. https:// doi.org/10.1118/1.4956728

11. Yuan Z, Rong Y, Benedict SH, Daly ME, Quu J, "Dose of the day" based on cone beam computed tomography and deformable image registration for lung cáncer radiotherapy, J Appl Clin Med Phys 2020;21(1):88-94 https://doi.org/10.1002/ acm2.12793

12. Bea-Gilabert J, Baños-Capilla MC, García-Martínez MA, López-Muñoz E, Larrea-Rabassa LM, An alternative approach to GTV margin determination in stereotactic body radiotherapy, J Radiosurg SBRT 2019;6(1):45-54 https://www.oldci- 
typublishing.com/journals/jrsbrt-home/jrsbrt-issue-contents/ journal-of-radiosurgery-sbrt-volume-6-number-1-2019/journal-of-radiosurgery-sbrt-6-1-p-45-54/

13. Sonke JJ, Rossi M, Wolthaus J, Van Herk M, Damen E, Belderbos J. Frameless stereotactic body radiotherapy for lung cancer using four- dimensional cone beam CT guidance, Int Jour Rad Onc Biol Phys 2009;74:567-74. https://doi. org/10.1016/j.ijrobp.2008.08.004

14. Van Herk M, Different styles of Image-guided Radiotherapy, Sem Rad Onc 2007;17:258-67 https://doi.org/10.1016/j. semradonc.2007.07.003

15. Kawrakow I, Fippel M, Friedrich K, 3D electron dose calculation using a Voxel based Monte Carlo algorithm (VMC), Med Phys 1996;23:445-57 https://doi.org/10.1118/1.597673

16. Fippel M, Fast Monte Carlo dose calculation for photon beams based on the VMC electron algorithm, Med Phys. 1999;26:1466-75. https://doi.org/10.1118/1.598676

17. Ahnesjo A, Collapsed cone convolution of radiant energy for photon dose calculation in heterogeneous media, Med Phys 1989;16(4):577-92 https://doi.org/10.1118/1.596360

18. Stambaugh C, Nelms BE, Dilling T, Stevens C, Latifi K, Zhang $G$ et al., Experimentally studied dynamic dose interplay dose not meaningfully affect target dose in VMAT SBRT lung treatments, Med Phys 2013;40(9):091710-1-8 http://dx.doi. org/10.1118/1.4818255
19. Andreo P, Dose to 'water-like' media or dose to tissue in MV photons radiotherapy treatment planning: still a matter of debate, Phys Med Biol 2015;60(1):309-37 https://doi. org/10.1088/0031-9155/60/1/309

20. Ma CM, Li J, Dose specification for radiation therapy: dose to water or dose to medium? Phys Med Biol 2011;56(10):307389 https://doi.org/10.1088/0031-9155/56/10/012

21. Siebers JV, Keall PJ, Nahum AE, Mohan R, Converting absorbed dose to medium to absorbed dose to water for Monte Carlo based photon beam dose calculations Phys Med Biol 2000;45(4):983-95 https://doi.org/10.1088/00319155/45/4/313

22. Rana S, Rogers K, Pokharel S, Cheng CY, Evaluation of Acuros XB algorithm based on RTOG 0313 dosimetric criteria for SBRT lung treatment with RapidArc, J Appl Clin Med Phys 2014;15(1):118-29 doi: 10.1120/jacmp.v15i1.4474

23. Archibald-Heeren BR, Byrne MV, Hu Y, Cai M, Wang $Y$, Robust optimization of VMAT for lung cancer: Dosimetric implications of motion compensation techniques, J Appl Clin Med Phys 2017;18(5):104-16 https://doi.org/10.1002/ acm2.12142

24. Unkelbach J, Alber M, Banger M, Bokrantz R, Chan TCY, $\mathrm{O}$ Deasy $\mathrm{J}$ et al., Robust radiotherapy planning, Phys Med Biol 2018;63(22)TR02 https://doi.org/10.1088/1361-6560/ aae659 


\section{¿En qué se basa nuestra innovación en iCT? En las necesidades de diagnóstico de más de $\mathbf{6 . 7 0 0}$ millones de personas.}

Del mismo modo que las enfermedades afectan a todos por igual, Brilliance iCT de Philips se adapta a todo tipo de pacientes. Adquiere imágenes nítidas de cualquier parte del cuerpo, desde lactantes de poco peso hasta adultos con

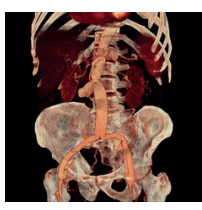

sobrepeso. Los pacientes recibirán el grado de calidad que se merecen en sus exploraciones mediante una dosis baja sin que ello afecte a la calidad de la imagen. Visite nuestra página web en www.philips.com/CT.

*Porque nuestras innovaciones se inspiran en usted.

PHILIPS

sense and simplicity

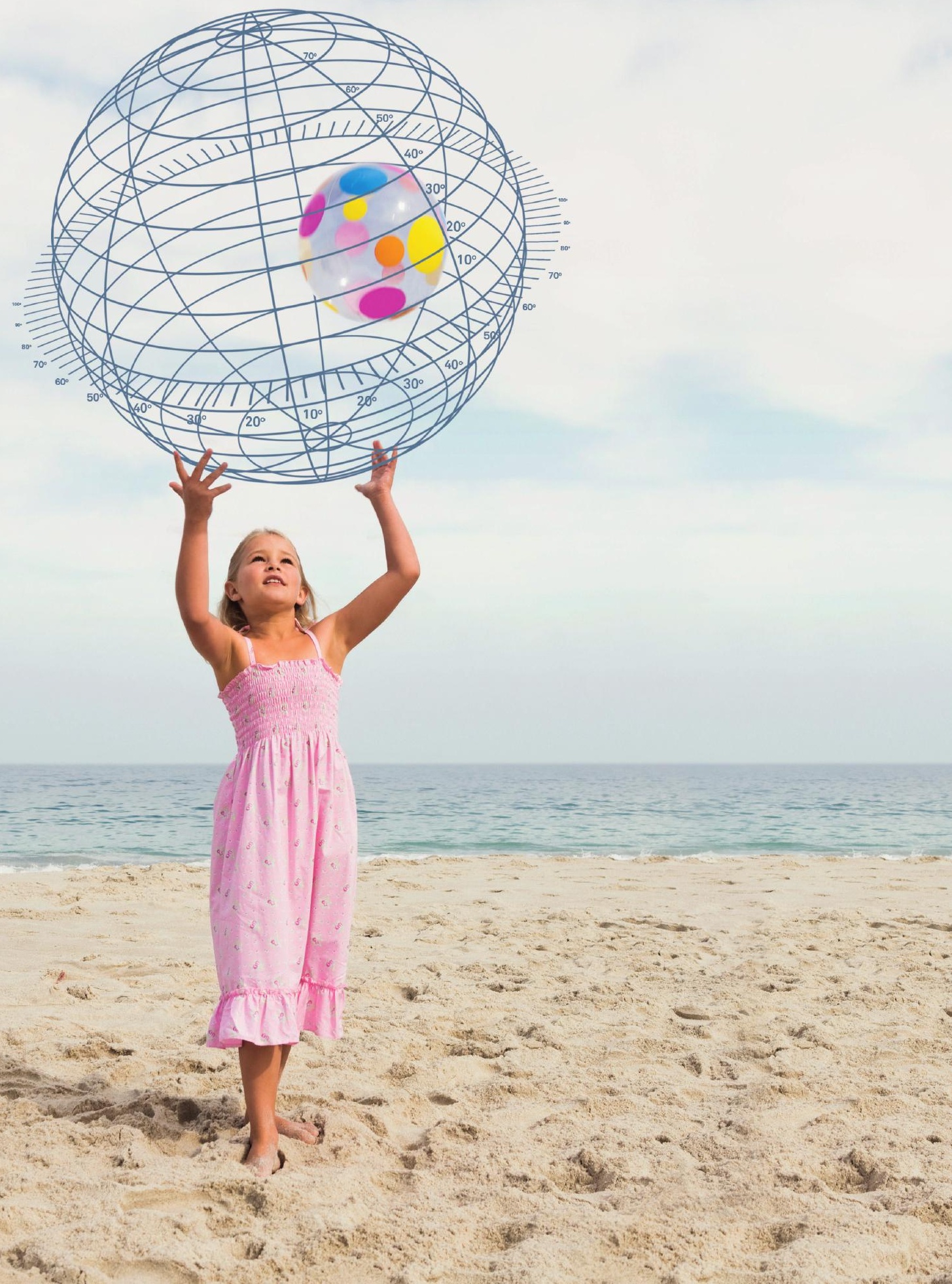

